\title{
Tweaking synchronization by connectivity modifications
}

\author{
Paul Schultz, ${ }^{1,2, *}$ Thomas Peron, ${ }^{1,3}$ Deniz Eroglu, ${ }^{1,2}$ Thomas Stemler, ${ }^{4}$ Gonzalo Marcelo Ramírez Ávila, ${ }^{5}$ \\ Francisco A. Rodrigues, ${ }^{6}$ and Jürgen Kurths ${ }^{1,2,7,8}$ \\ ${ }^{1}$ Potsdam Institute for Climate Impact Research, P.O. Box 6012 03, 14412 Potsdam, Germany \\ ${ }^{2}$ Department of Physics, Humboldt University of Berlin, Newtonstrasse 15, 12489 Berlin, Germany \\ ${ }^{3}$ Instituto de Física de São Carlos, Universidade de São Paulo, São Carlos, São Paulo, Brazil \\ ${ }^{4}$ School of Mathematics and Statistics, The University of Western Australia, 35 Stirling Highway, Crawley, WA 6009, Australia \\ ${ }^{5}$ Instituto de Investigaciones Físicas, Casilla 8635, Universidad Mayor de San Andrés, La Paz, Bolivia \\ ${ }^{6}$ Instituto de Ciências Matemáticas e de Computaçao, Universidade de São Paulo, CP 668, 13560-970 São Carlos, São Paulo, Brazil \\ ${ }^{7}$ Institute for Complex Systems and Mathematical Biology, University of Aberdeen, Aberdeen AB24 3UE, United Kingdom \\ ${ }^{8}$ Department of Control Theory, Nizhny Novgorod State University, Gagarin Avenue 23, 606950 Nizhny Novgorod, Russia
}

(Received 24 March 2016; published 10 June 2016)

\begin{abstract}
Natural and man-made networks often possess locally treelike substructures. Taking such tree networks as our starting point, we show how the addition of links changes the synchronization properties of the network. We focus on two different methods of link addition. The first method adds single links that create cycles of a well-defined length. Following a topological approach, we introduce cycles of varying length and analyze how this feature, as well as the position in the network, alters the synchronous behavior. We show that in particular short cycles can lead to a maximum change of the Laplacian's eigenvalue spectrum, dictating the synchronization properties of such networks. The second method connects a certain proportion of the initially unconnected nodes. We simulate dynamical systems on these network topologies, with the nodes' local dynamics being either discrete or continuous. Here our main result is that a certain number of additional links, with the relative position in the network being crucial, can be beneficial to ensure stable synchronization.
\end{abstract}

DOI: 10.1103/PhysRevE.93.062211

\section{INTRODUCTION}

The study of dynamical processes on complex networks has been one of the most active fields within network science, where the ultimate goal is the precise evaluation of how network topology affects dynamics [1]. The first numerical and analytical approaches to this problem were mainly concerned with the pure effect of the heterogeneity of the degree distribution on the overall network dynamics [1]. A big reason for the interest in this subject has been the detailed description of the topology of real systems that the network representation offers, a fact that motivates the search for a better understanding of their dynamical behavior as well. Early analytical developments, therefore, were mostly based on the configuration model [2], which is able to construct networks with a given degree distribution. While offering a benchmark to study dynamical processes in networks with any kind of degree distribution, the networks constructed using such a model are generally locally treelike, i.e., with a vanishing density of cycles [3]. On the other hand, it is known that real networks in turn exhibit a much more sophisticated topology, which encompasses features such as clustering, high-order loops, degree-degree correlations, community organization, etc. [3]. Thus, it is evident that studies based solely on the configuration model oversee topological characteristics that are essential for a thorough analysis of the function of real networks.

To overcome this limitation and take into account higherorder topological features, two strategies are commonly used,

\footnotetext{
*pschultz@pik-potsdam.de
}

namely the adoption of networks constructed through stochastic rewiring algorithms [4-6], or variations of the traditional configuration model that allow the creation of networks with tunable clustering or other kinds of subgraphs [7-9]. In the former, one starts with a random treelike network and switches the edges according to some heuristics in order to obtain a desirable network configuration, which is then used as a substrate for the dynamics under study. Although this approach enables precise control of a given network property, as a function of which the dynamics can be analyzed, other properties are dramatically changed [4-6]. The latter makes the assessment of the isolated contribution of a particular topological property to the network dynamics unfeasible, since the results can be potentially influenced by spurious effects generated by the method. This limitation can be surpassed by extensions of the configuration model. However, depending on the subgraph structure modeled, the computational complexity quickly escalates, imposing further constraints on the analysis $[8,9]$.

In this paper, we address the effect of particular structural patterns found in real networks, namely cycles of different lengths, by adopting a different approach. To control their occurrence, and therefore evaluate their contribution to the network dynamics, here we consider tree networks with minimal link addition in such a way that the number and the length of the cycles are precisely varied. In addition to the thorough control of network structures being created, the minimal link addition adopted here paves the way for new strategies intended to enhance the stability of real networks. The reason for that resides in the fact that the creation and rewiring of links are usually costly tasks to be performed in these systems [10]. 
A prominent example is that of power grids, whose proper functioning is vital for modern society. Since the connectivity pattern of the surroundings of a given dynamical unit strongly influences its stability [11-14], it is crucial that the inclusion of new transmission lines is done in such a way to ensure, or even enhance, the local and global stability of the network, while spending minimal amounts of resources. Otherwise, counterintuitive dynamical effects such as Braess' paradox lead to certain new links destabilizing synchronization by increasing the largest Lyapunov exponent, eventually changing its sign [11,13]. This is related largely to the appearance of cycles in the network. Similar arguments also hold for other spatially embedded man-made or natural networks with constrained connectivity, for instance transportation or neuronal networks.

This paper contributes in this direction by quantifying the impact of cycles created under minimal link addition on the global network behavior with a focus on stability. In general, however, we expect that our approach translates to a broad class of problems ranging from synchronization to percolation, spreading processes $[15,16]$, or control theory [17]. Regarding the network dynamics, we consider the nodes as identical oscillators operating in periodic or chaotic regimes in the paradigmatic cases of logistic maps and Rössler oscillators. We evaluate the stability of the synchronous regimes depending on the variation of the length of cycles in the network topology. By employing the master stability function (MSF) formalism [18], we map the problem into a spectral analysis of the Laplacian matrix. This spectral approach has also been successfully applied to reveal network-dependent coherence [19]. Recently, Pade and Pereira showed that link additions in directed networks can destabilize the synchronous regime [20]. Furthermore, in the case of a removal of links or altered link weights, changes to the synchronous state and its stability are also found to relate to the Laplacian spectrum [14], in leading order to the Fiedler eigenvector. In this paper, we study undirected networks and link addition, considering the whole stability interval of coupling values instead of only the lower boundary.

Our results suggest that cycles of length 4 play a special role in network dynamics. More precisely, we find that the inclusion of links that create these motifs yields networks with higher synchronizability in comparison with cycles of different lengths. Furthermore, cycles of length 3 are found to have a weak effect on the Laplacian spectrum, and, consequently, on the stability of the synchronized state. Interestingly, this peculiar innocuous effect of triadic connections on critical dynamical properties has also been reported in other contexts [21-25].

This paper is organized as follows: In the next section, we explain how we create different network topologies starting from a tree. We present two different methods: (i) introducing only one cycle of a given length, and (ii) adding several random links. Moreover, we show in detail how different cycle lengths change the properties of the network that control the synchronizability. Thereafter, we study how the number of random links added to the starting tree impacts the synchronization of the network using two numerical models, namely the time discrete logistic map and the continuous Rössler system. Finally, we state the conclusions and perspectives.

\section{NETWORK TOPOLOGIES}

\section{A. Network manipulation}

The network generation procedure is as follows. As a starting point, we consider an undirected balanced tree graph $G(m, n)$, where $m$ is the number of levels and $n$ is the branching number at each level. Therefore, the total number of nodes is given by $N=\left(n^{(m+1)}-1\right) /(n-1)$ and the total number of edges is $N-1$. Due to the fact that the total number of edges in a complete undirected graph is $N(N-1) / 2$, the remaining unconnected number of pairs for the tree structure is given by $r=(N-2)(N-1) / 2$. Notice that our starting network does not have any cycles.

Having a highly structured-treelike-network with known statistical characteristics allows us to control the network topology in two different ways. One is targeting the network topology directly by introducing cycles of a chosen length. The other way lets us evaluate what happens if we control the sparseness of the network by randomly introducing a certain amount of additional edges. We are going to refer to the introduction of cycles as the single link addition. Here the length of the cycle is the control parameter. The other method will be referred to as random link addition, and in this case the control parameter is the probability $p$ multiplying the number of unconnected pairs $r$. We want to point out that this network generation method is very similar to those used to generate small-world structures [26]. However, the substantial difference is that our initial network is a balanced tree structure instead of a regular lattice.

What we aim to evaluate is how the different lengths of cycles and the choice of $p$ values change the synchronization features of the networks. We consider the resulting network structure of our two methods as the adjacency matrix $A_{i j}$, with $A_{i j}=1$ if nodes $i$ and $j$ are connected, and $A_{i j}=0$ otherwise. The number of connections, the degree, of node $i$ is given by $k_{i}=\sum_{j} A_{i j}$. Further, we define the Laplacian $\mathbf{L}, L_{i j}=$ $\delta_{i j} k_{i}-A_{i j}$. Its eigenvalues $\lambda_{i}\left(\lambda_{1}=0 \leqslant \lambda_{2} \leqslant \cdots \leqslant \lambda_{\text {max }}\right)$ play an important role in characterizing the synchronizability of the system and therefore measure comprehensively what we want to determine [18], i.e., changes in the eigenvalue spectrum of our designed networks. In particular, we are interested in the minimal changes or best cycle lengths that have maximum impact on the value of $\lambda_{2}$ or $\lambda_{\max }\left(\lambda_{1}=0\right.$ if the network is connected). The magnitude of the first nontrivial eigenvalue $\lambda_{2}$ is related to the onset of synchronization, while the magnitude of the maximum eigenvalue $\lambda_{\max }$ is connected to the end of the synchronization interval $[3,27]$.

\section{B. Impact of cycle length}

While we are going to study the random link addition later when focusing on network dynamics, we start by studying just the eigenvalue pair $\lambda_{2}$ and $\lambda_{\max }$ as a function of the cycle length after single link addition. Avoiding self-loops, the first cycle is a 3-cycle, i.e., a node's neighbors in the network are themselves connected. Using the illustration in Fig. 1 of a balanced tree network $G(m, 3)$ (only $m=3$ levels are shown), we see that introducing a 3 -cycle in this network means connecting two nodes $i$ and $j$ that lie on one of the circles highlighting the level number (see the dashed blue highlighted line in Fig. 1). 


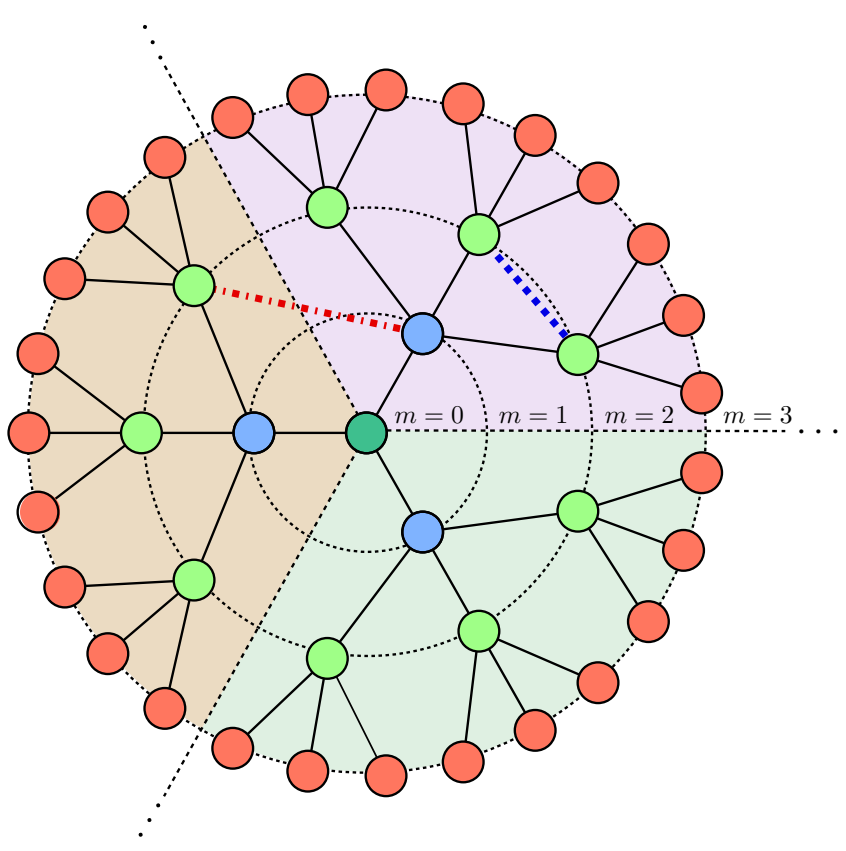

FIG. 1. Sketch of a balanced tree network $G(m, 3)$. In the single link addition procedure, we distinguish two cases, namely connecting nodes in the same fundamental branch ("in," _. ) ) or in different ones("out," - - ), i.e., every path between the nodes before the addition contains the root node.

In addition, note that the branching on level $m=1$ of the original network is highlighted as the shading of the three areas. Ignoring the trivial level $m=1$, all of the 3-cycles that we can create lie within the same shaded area and do not break the symmetry of the network. This also means that they are linking two nodes within the same branch of the tree network (shaded areas with the same color in Fig. 1), and we call these links in-links. On the other hand, if a link connects nodes belonging to different branches (e.g., the dash-dotted red line in Fig. 1), we denote it as an out-link. In this case, the shortest cycle created by an out-link is a 4-cycle.

In Fig. 2(a), we can see how distinguishing between inand out-links can be used as a tool to generate cycles of different lengths [shown for $G(5,3)]$. Starting from the center of the network, we number all nodes and their levels based on their distance. In this matrixlike plot we find that, for example, linking two nodes in the first level (1-out) creates a 3-cycle. Similarly, a 3-cycle can be created by linking from the second level (2-out) back to the center (0-out). As we can see in $G(5,3), 11$-cycles are the longest possible cycles in this network. Note also that the average path length is changed by the introduction of different cycles. Especially short cycles will impact the average shortest path length, while long cycles, e.g., out-links connecting leaf nodes, are likely to only have a small effect.

Since we are focusing on changes of the Laplacian's eigenvalues, it is worth pointing out that adding links in any undirected network always positively increases the magnitude of these eigenvalues. From a synchronization point of view, for instance, we are interested in the maximum possible change $\Delta \lambda_{\max }$ of the maximum Laplacian eigenvalue. In Fig. 2, we show how much the different cycles change the magnitude

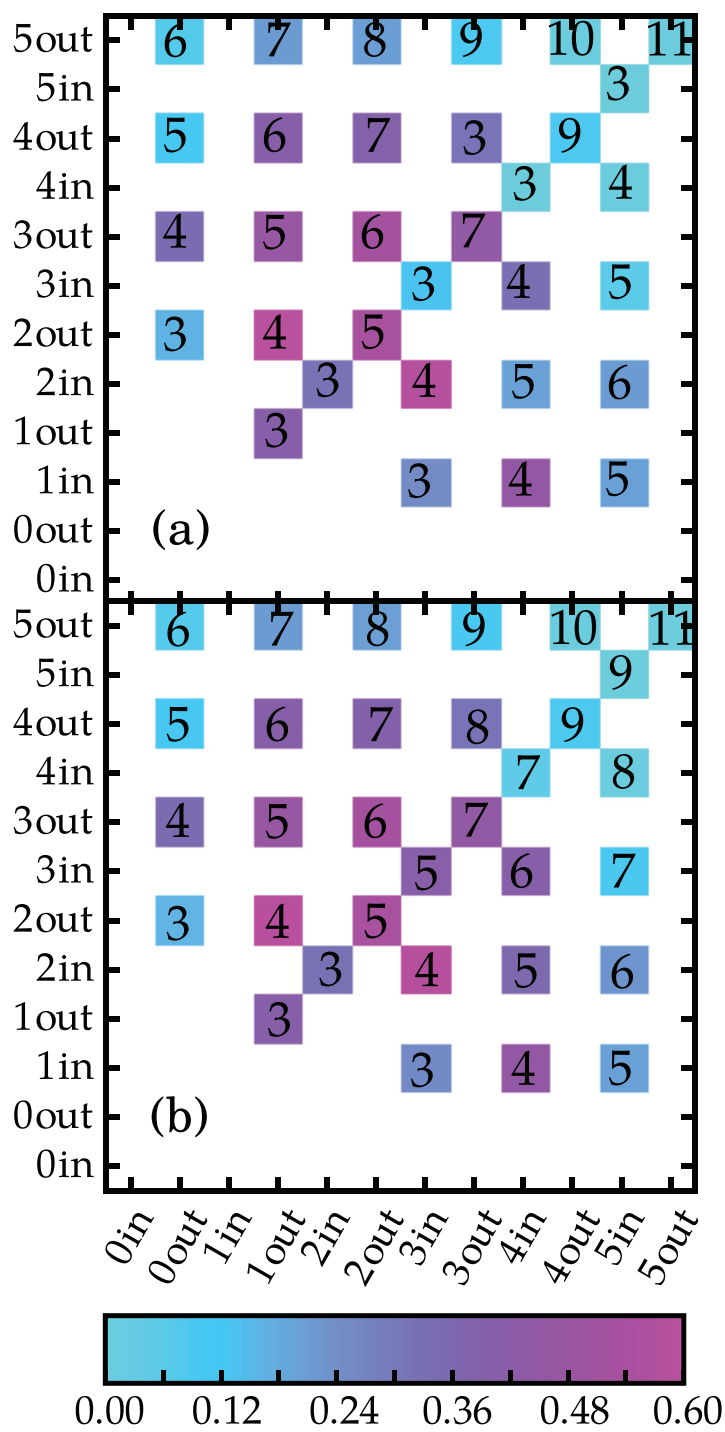

FIG. 2. (a) Minimal and (b) maximal increase $\Delta \lambda_{\max }$ of the largest Laplacian eigenvalue $\lambda_{\max }$ for various wiring choices. For reference, the integers denominate the length of the (a) shortest and (b) longest cycle possible for each configuration. For the link classification "in" vs “out," see Fig. 1.

of $\lambda_{2}$ and $\lambda_{\max }$. Again, we present these changes in terms of in- and out-level connections. We want to highlight some of these changes. First, we note that of the possible 3-cycles, the one with the strongest impact on the eigenvalues is on $m=1$, while $m \geqslant 2$ has less of an impact. In particular, connecting to neighboring leaf nodes in level 5 does not change the upper limit of the spectrum, while the largest sensitivity to topological changes is observed close to the root node. This tendency is repeated again in the 4-cycles, where the strongest impact is found when 2-out is connected to a note on $m=1$ while already connections from 3-out to $m=2$ do not lead to such a large change. For the 3- and 4-cycles, we summarize this topological effect in Fig. 3.

If we ignore the additional information on where the cycle is within the network, it is valid to ask about the general impact of the cycle's length on changes in the eigenvalues. This information can be found in Fig. 4 for the distribution of changes in the largest Laplacian eigenvalue of $G(5,3)$. It is not surprising that 

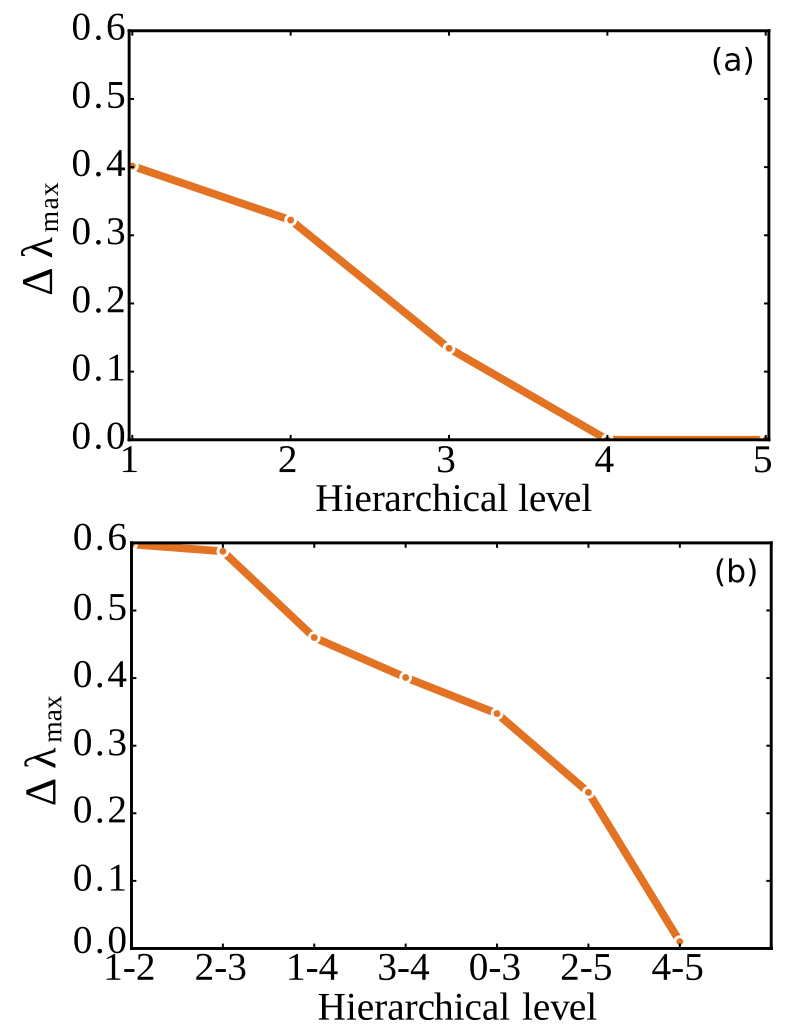

FIG. 3. Increase of the maximum Laplacian eigenvalue $\lambda_{\max }$ for connections (a) on the same level creating cycles of length 3 and (b) between different levels creating cycles of length 4 .

long cycles play almost no role in small-sized networks, while 4-cycles are dominating the change. Furthermore, 3-cycles induce changes in the spectrum comparable to long cycles, suggesting that such topological patterns have a weak impact on network dynamics as well. This is somewhat remarkable given how pervasive 3-cycles are in real-world networks [3]. It was verified (not shown here) that changes in $\Delta \lambda_{\max }$ due to the share of long cycles increase with the system size, but 4-cycles are still dominating. 3-cycles were consistently found to be responsible for smaller changes in the spectrum than 4-cycles. This has also been addressed by Lodato et al. comparing the

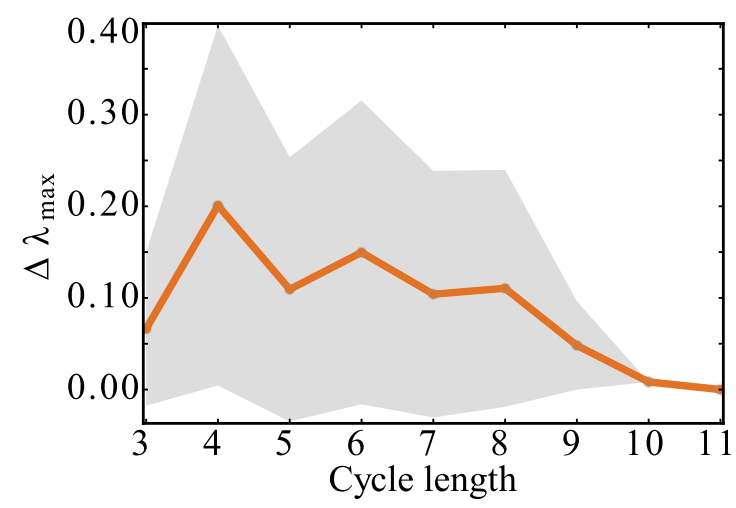

FIG. 4. The average change of the maximum Laplacian eigenvalue is given as bullets while the shaded area indicates the width of the distribution between the minimal and the maximal change within the possible cycles in the network.

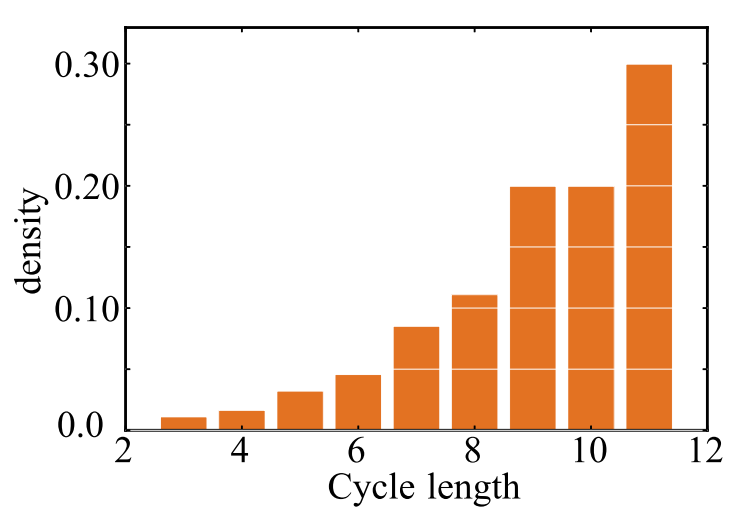

FIG. 5. Relative occurrence of cycle lengths when randomly connecting two nodes in a $G(5,3)$ balanced tree.

synchronizability of 4-node subgraphs, quantified by the ratio $\lambda_{\max } / \lambda_{2}$ of the Laplacian eigenvalues [28]. Their analysis showed that 4-node motifs containing a single 3-cycle have lower synchronizability than motifs with the same number of nodes and links containing a 4-cycle. Interestingly, a single link addition that creates two 3-cycles in a 4-node motif does not increase its synchronizability. Therefore, their result suggests that networks with a higher number of 4-cycles are more prone to exhibit a synchronized state.

Concerning the random link addition, it is of great interest to estimate the probability for cycles of a particular length being created. As we can see from the relative occurrence of cycle lengths plotted in Fig. 5, it is more likely that we choose longer cycles, i.e., on average a randomly chosen link would create a 9-cycle. Therefore, we can expect that large changes associated, for example, with some of the 4-cycles will be less likely to dominate the eigenvalue spectrum, while the sum of changes resulting from several longer cycles will make up the main effect that changes the eigenvalue spectrum.

\section{MASTER STABILITY FUNCTION}

By means of the above-mentioned methods, we obtain the adjacency matrices of the networks containing our prototypical dynamic systems. We investigate two paragons of dynamical systems theory, namely Rössler oscillators, as an example of an autonomous continuous system, and the well-established logistic map as a discrete one. Our main tool allowing us to find relationships between the network spectral properties and the stability of synchronous regimes of the considered dynamical systems is the master stability function (MSF) approach [29].

To illustrate the method, we assume first a continuous (autonomous) dynamical system of, for instance, coupled oscillators described by a state vector $\mathbf{x}_{i}$ at site $i$ in a network:

$$
\dot{\mathbf{x}}_{i}=\mathbf{F}\left(\mathbf{x}_{i}\right)+\sigma \sum_{j=1}^{N} A_{i j} \mathbf{H}\left(\mathbf{x}_{i}, \mathbf{x}_{j}\right),
$$

where $\mathbf{F}$ is the individual oscillator's dynamics, $\mathbf{H}$ is the coupling function between coupled elements, $A_{i j}$ is an element of the network's adjacency matrix, and $\sigma$ is the overall coupling strength. To assess whether our networks allow for stable solutions of complete synchronization, i.e., $\mathbf{x}_{i} \equiv \mathbf{s} \forall i=$ $1, \ldots, N$, we need to obtain the MSF. For identical oscillators, 
this function is given by [29]

$$
\operatorname{MSF}_{\mathbf{F}, \mathbf{H}}\left(\alpha_{i}\right)=D \mathbf{F}(\mathbf{s})-\alpha_{i} D \mathbf{H}(\mathbf{s}),
$$

where $\alpha_{i}=\sigma \lambda_{i}$ with Laplacian eigenvalues $\lambda_{i}$, and $D \mathbf{F}$ and $D \mathbf{H}$ are the Jacobian of the system and of the coupling function. If we assume for a moment that the argument $\alpha$ is a continuous (in general complex) variable, the real roots of the maximum Lyapunov exponent $\Lambda_{\text {max }}$ of $\operatorname{MSF}_{\mathbf{F}, \mathbf{H}}(\alpha)$ determine the boundaries $\alpha_{l}$ and $\alpha_{u}$ of the stability interval. Note that for periodic systems, there is only one $\operatorname{root} \alpha_{u}$ as $\alpha_{l}=0$. To have a stable solution, the coupling $\sigma$ needs to be chosen in such a way that $\alpha_{l}<\alpha_{i}<\alpha_{u} \forall i=1, \ldots, N$ for $\Lambda_{\max }$ to be negative and hence to have an asymptotically stable synchronous regime (cf. Fig. 6).

As discussed in the preceding section, the most crucial nontrivial Laplacian eigenvalues to be fit in the stability interval are the second minimum $\lambda_{2}$ and the largest $\lambda_{\max }$ ones, since the rest of the eigenvalues are distributed between them.

In the following, we apply this approach to the Rössler system as oscillatory units that are coupled through their $x$ coordinates:

$$
\begin{aligned}
& \dot{x}_{i}=-y_{i}-z_{i}+\sigma \sum_{j=1}^{N} A_{i j}\left(x_{j}-x_{i}\right), \\
& \dot{y}_{i}=x_{i}+a y_{i}, \\
& \dot{z}_{i}=b+z_{i}\left(x_{i}-c\right) .
\end{aligned}
$$
by

Computing $\operatorname{MSF}_{\mathbf{F}, \mathbf{H}}(\alpha)$, we find that $D \mathbf{F}$ and $D \mathbf{H}$ are given

$$
D \mathbf{F}=\left(\begin{array}{ccc}
0 & -1 & -1 \\
1 & a & 0 \\
z^{*} & 0 & x^{*}-c
\end{array}\right), \quad D \mathbf{H}=\left(\begin{array}{ccc}
1 & 0 & 0 \\
0 & 0 & 0 \\
0 & 0 & 0
\end{array}\right) .
$$
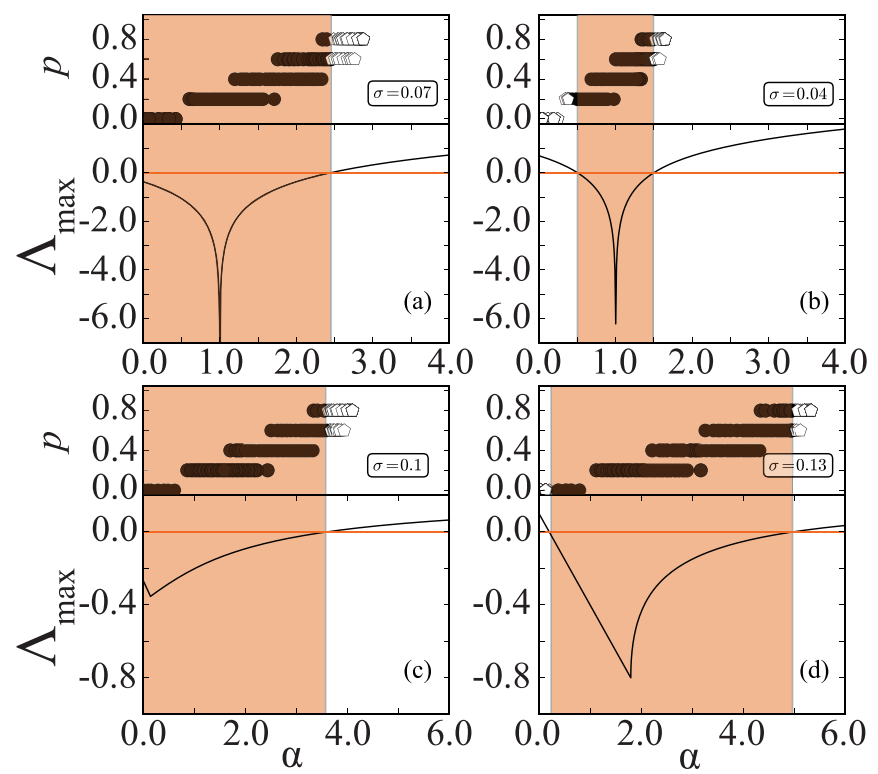

FIG. 6. Coupled logistic map in (a) periodic and (b) chaotic regimes, and Rössler oscillators in (c) periodic and (d) chaotic regimes. The upper plots depict the nonzero eigenvalues of the Laplacian $\mathbf{L}$ for different probability $p$ of link addition (see the text for details). The shaded area denotes the existence of stable synchronous solutions. In all panels, the initial topology was $G(3,3)$.
For our investigations, we choose the parameters such that we have one set in the periodic regime $(a=0.1, b=0.2$, and $c=5.7)$ and one in the chaotic regime $(a=0.2, b=0.2$, and $c=5.7$ [30]). Notice that the MSF in general only yields a valid linearization in a neighborhood around a point $\left(x^{*}, y^{*}, z^{*}\right)^{T}$ from the synchronization manifold, which we determine from numerical simulations of the system. We obtain $\alpha_{u}=9.99884$ and $\left(\alpha_{l}, \alpha_{u}\right)=(0.198769,4.99878)$ for the periodic and chaotic cases, respectively.

As a second application, we use the time-discrete logistic map as an oscillator given by

$$
\begin{gathered}
x_{t+1}^{i}=(1-\sigma) f\left(x_{t}^{i}\right)+\frac{\sigma}{k_{i}} \sum_{j} A_{i j}\left[f\left(x_{t}^{i}\right)-f\left(x_{t}^{j}\right)\right], \\
f\left(x_{t}^{i}\right)=r x_{t}^{i}\left(1-x_{t}^{i}\right),
\end{gathered}
$$

where the local node dynamics $f\left(x_{t}^{i}\right)$ is the logistic map ( $r \in$ $[0,4]), \sigma \in[0 ; 1]$ is the coupling strength between the units, and $A$ is an adjacency matrix.

The logistic map is one-dimensional, therefore the Jacobian of Eq. (5) is the derivative of $f$ with respect to $x_{i}$, and the associated MSF is given by

$$
\operatorname{MSF}_{f}\left(\alpha_{i}\right)=\left(1-\alpha_{i}\right) f^{\prime}\left(x_{t}^{i}\right),
$$

where $\alpha_{i}=\sigma \lambda_{i} ; \lambda_{i}$ are the eigenvalues of the Laplacian, as stated above.

For our research, we choose the control parameter of the logistic map, $r$, such that we have one periodic case $(r=3.83$, period 3) and one chaotic case $(r=4.0)$. For the periodic case, $\Lambda_{\max }$ again has only one root, which is found to be $\alpha_{u}=$ 2.45157 (Fig. 6). The roots for the chaotic local dynamics are found to be $\alpha_{l}=0.50038$ and $\alpha_{u}=1.49962$.

We demonstrate the stability of the coupled oscillators representing the nodes of our networks. We study the synchronization behavior of two different node types: the nodes' dynamics are given either by a logistic map or the continuous Rössler system. Both systems are analyzed in the periodic and chaotic dynamical regimes. As we can see in Fig. 7, the eigenvalues $\lambda_{2}$ and $\lambda_{\max }$ change with a different rate depending on the parameter $p$, which controls the random link addition. While the initial growth of $\lambda_{\max }$ dominates for low $p$-values, it seems to saturate for high values where we observe most of the change for $\lambda_{2}$.

Hence, if just a few links are added at random locations, the initial growth of $\lambda_{\max }$ leads to a spreading of the Laplacian spectrum, with the potential to cross the upper limit $\alpha_{u}$ of the synchronization interval. This can be counteracted by lowering the coupling $\sigma$, i.e., small changes in the network should be accommodated by reducing $\sigma$ to safely pertain in the synchronous interval.

In summary, Fig. 6 depicts the qualitative results for MSFs of the two test systems; the shaded areas denote the synchronous region. In the white regions, the synchronous regime is unstable. As mentioned above, increasing $p$-values leads to an increase in the edges, and similarly the Laplacian eigenvalues increase as well.

(i) The lower boundary for the periodic cases is $\alpha_{l}=0$ [cf. Figs. 6(a) and 6(c)], so that one only needs to consider the upper limit to ensure stable synchronization. 


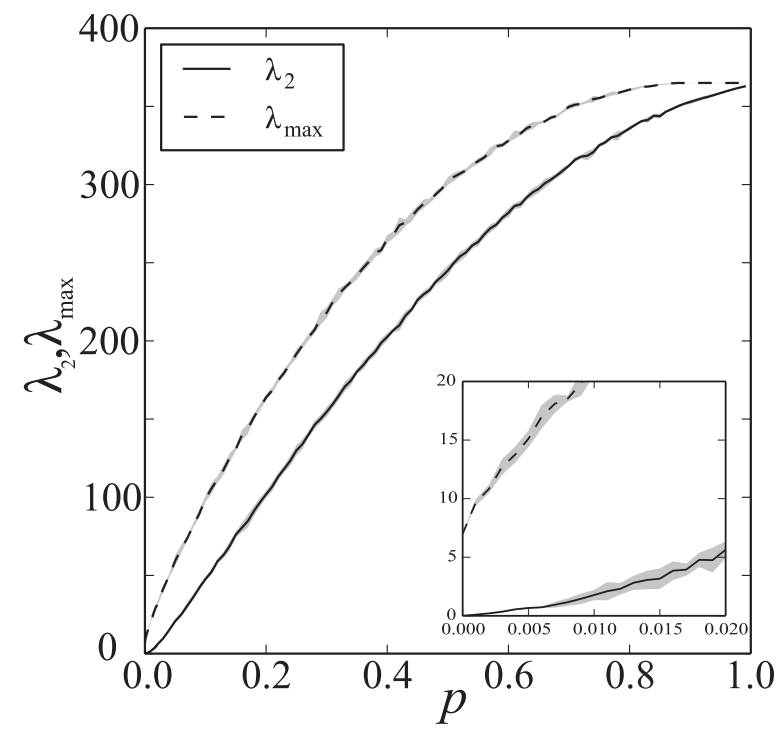

FIG. 7. Pictured are the average eigenvalues $\lambda_{2}$ and $\lambda_{\max }$ for varying linkage probability $p$; the shading indicates one standard deviation. The inset magnifies the regime of $0<p<0.02$. Note that the two eigenvalues $\lambda_{2}$ and $\lambda_{\max }$ bound the eigenvalue spectrum of the Laplacian.

(ii) The lower boundary of the chaotic case is positive. Therefore, both limits must be considered [cf. Figs. 6(b) and 6(d)].

As we can see in all four cases, there is a maximum number of links that we can add before the system reaches the upper threshold of the synchronization region. Clearly, if we choose $p=0.8$, some $\alpha$ values are no longer within the shaded synchronization regions in all panels of Fig. 6 (see the highlighted pentagons above each graph). More interesting is the situation for the oscillators being chaotic [cf. Figs. 6(b) and $6(\mathrm{~d})]$. We can see that without adding any links $(p=0)$, the network does not support synchronization. Increasing the number of links leads to networks that can support synchronization, but again too many links will increase the eigenvalues by too much, and we cannot find synchronization anymore. Comparing these results with Fig. 7, we see that $p$ needs to be big enough to increase $\lambda_{2}$ above the level where the lower bound is already in the synchronization region, while $\lambda_{\max }$ is still below the upper threshold of the band.

\section{CONCLUSION}

Since large natural or man-made networks are locally treelike, we have focused on small trees as the starting point of constructing our network. We have seen that even including one edge can substantially alter the synchronization behavior of the system, and that short cycles connecting different levels of the tree have the largest impact on the eigenvalues of the Laplacian, especially if they are created close to the root node. We have highlighted a way to add cycles of defined length in our trees, which gives us the option to design networks having a particular synchronization behavior.

Using the master stability framework, we were able to analyze how random link addition alters the synchronization phenomenon using the logistic map as well as the Rössler oscillator. The most striking example that we studied is the synchronization behavior of nodes having chaotic dynamics. While without additional links the networks are unable to synchronize the dynamics of the nodes, adding some links to the networks alters the global dynamics essentially, and the systems can synchronize. Moreover, we have found that adding too many links causes desynchronization.

In conclusion, our work provides a method to optimize networks in such a way that they can become synchronized in an improved manner. Given that synchronization is of the utmost importance in many networks, e.g., power grids [12,25,31,32], neuronal networks $[25,33,34]$, and communication networks [34], our findings may be used to increase the stability of the synchronization regime by adding short cycles.

\section{ACKNOWLEDGMENTS}

The authors wish to thank the Nesin Foundation for an amazing working group activity in Nesin Math Village, and we wish to thank Tiago Pereira for fruitful discussions. P.S. and J.K. acknowledge gratefully the support of BMBF, CoNDyNet, FK. 03SF0472A. T.P. acknowledges FAPESP (No. 2012/22160-7 and No. 2015/02486-3) and IRTG 1740. D.E. acknowledge support by the Leibniz Association (WGL) under Grant No. SAW-2013-IZW-2542.
[1] A. Barrat, M. Barthelemy, and A. Vespignani, Dynamical Processes on Complex Networks (Cambridge University Press, Cambridge, 2008).

[2] M. E. J. Newman, S. H. Strogatz, and D. J. Watts, Phys. Rev. E 64, 026118 (2001).

[3] M. Newman, Networks: An Introduction (Oxford University Press, Oxford, 2010).

[4] B. J. Kim, Phys. Rev. E 69, 045101 (2004).

[5] R. Xulvi-Brunet and I. M. Sokolov, Phys. Rev. E 70, 066102 (2004).

[6] D. M. Green and I. Z. Kiss, J. Biol. Dyn. 4, 431 (2010).

[7] M. E. J. Newman, Phys. Rev. Lett. 103, 058701 (2009).

[8] B. Karrer and M. E. J. Newman, Phys. Rev. E 82, 066118 (2010).
[9] V. Zlatić, D. Garlaschelli, and G. Caldarelli, Europhys. Lett. 97, 28005 (2012).

[10] M. Barthélemy, Phys. Rep. 499, 1 (2011).

[11] T. Coletta and P. Jacquod, Phys. Rev. E 93, 032222 (2016).

[12] P. J. Menck, J. Heitzig, J. Kurths, and H. J. Schellnhuber, Nat. Commun. 5, 3969 (2014).

[13] D. Witthaut and M. Timme, New J. Phys. 14, 083036 (2012).

[14] D. Witthaut, M. Rohden, X. Zhang, S. Hallerberg, and M. Timme, Phys. Rev. Lett. 116, 138701 (2016).

[15] A. Pikovsky, M. Rosenblum, and J. Kurths, Synchronization: A Universal Concept in Nonlinear Sciences (Cambridge University Press, Cambridge, 2003), Vol. 12, p. 3. 
[16] J. D. Noh and H. Rieger, Phys. Rev. Lett. 92, 118701 (2004).

[17] V. M. Preciado, M. M. Zavlanos, A. Jadbabaie, and G. J. Pappas, in American Control Conference (ACC) (IEEE, Piscataway, NJ, 2010), pp. 4462-4467.

[18] M. Barahona and L. M. Pecora, Phys. Rev. Lett. 89, 054101 (2002).

[19] T. Pereira, D. Eroglu, G. B. Bagci, U. Tirnakli, and H. J. Jensen, Phys. Rev. Lett. 110, 234103 (2013).

[20] J. P. Pade and T. Pereira, Sci. Rep. 5, 9968 (2015).

[21] S. Melnik, A. Hackett, M. A. Porter, P. J. Mucha, and J. P. Gleeson, Phys. Rev. E 83, 036112 (2011).

[22] S. Yoon, A. V. Goltsev, S. N. Dorogovtsev, and J. F. F. Mendes, Phys. Rev. E 84, 041144 (2011).

[23] C. P. Herrero, Phys. Rev. E 91, 052812 (2015).

[24] T. K. D. Peron, F. A. Rodrigues, and J. Kurths, Phys. Rev. E 87, 032807 (2013).
[25] F. A. Rodrigues, T. K. D. Peron, P. Ji, and J. Kurths, Phys. Rep. 610, 1 (2016).

[26] M. Newman and D. Watts, Phys. Lett. A 263, 341 (1999).

[27] S. Boccaletti, V. Latora, Y. Moreno, M. Chavez, and D. Hwang, Phys. Rep. 424, 175 (2006).

[28] I. Lodato, S. Boccaletti, and V. Latora, Europhys. Lett. 78, 28001 (2007).

[29] L. M. Pecora and T. L. Carroll, Phys. Rev. Lett. 80, 2109 (1998).

[30] O. E. Rössler, Phys. Lett. 57A, 397 (1976).

[31] M. Rohden, A. Sorge, M. Timme, and D. Witthaut, Phys. Rev. Lett. 109, 064101 (2012).

[32] A. E. Motter, S. A. Myers, M. Anghel, and T. Nishikawa, Nat. Phys. 9, 191 (2013).

[33] E. Bullmore and O. Sporns, Nat. Rev. Neurosci. 10, 186 (2009).

[34] A. Arenas, A. Díaz-Guilera, J. Kurths, Y. Moreno, and C. Zhou, Phys. Rep. 469, 93 (2008). 